\title{
Detection of fast and slow crystallization processes in instantaneously-strained samples of cis-1,4-polyisoprene
}

\section{$\operatorname{AUTHOR}(\mathrm{S}):$}

Tosaka, Masatoshi; Senoo, Kazunobu; Sato, Kenta; Noda, Miki; Ohta, Noboru

\section{CITATION:}

Tosaka, Masatoshi ... [et al]. Detection of fast and slow crystallization processes in instantaneously-strained samples of cis-1,4-polyisoprene. Polymer 2012, 53(3): 864-872

\section{ISSUE DATE:}

2012-02

URL:

http://hdl.handle.net/2433/153409

\section{RIGHT:}

(C) 2011 Elsevier Ltd.; This is not the published version. Please cite only the published version.; この論文は出版社版でありません。引用の際に は出版社版をご確認ご利用ください。 


\title{
Detection of Fast and Slow Crystallization Processes
}

\section{in Instantaneously-Strained Samples of cis-1,4-}

\section{polyisoprene}

\author{
Masatoshi Tosaka, ${ }^{\mathrm{a}, *}$ Kazunobu Senoo, ${ }^{\mathrm{b}}$ Kenta Sato, ${ }^{\mathrm{b}}$ Miki Noda, ${ }^{\mathrm{b}}$ Noboru Ohta ${ }^{\mathrm{c}}$ \\ ${ }^{a}$ Institute for Chemical Research, Kyoto University, Gokasho, Uji, Kyoto 611-0011, Japan \\ ${ }^{\mathrm{b}}$ Kobe Fundamental Research Laboratory, Sumitomo Bakelite Co., Ltd., 1-1-5 Murotani, Nishi- \\ ku, Kobe, Hyogo 651-2241, Japan
}

'SPring-8 / JASRI, 1-1-1 Kouto, Sayo-cho, Sayo-gun, Hyogo 679-5198, Japan

E-mail: tosaka@scl.kyoto-u.ac.jp (M. Tosaka), senoo@sumibe.co.jp (K. Senoo), sato@sumibe.co.jp (K. Sato), m-noda@sumibe.co.jp (M. Noda), noboru_o@spring8.or.jp (N. Ohta)

* Corresponding Author: E-mail tosaka@scl.kyoto-u.ac.jp; Ph +81-774-38-3062; Fax +81-774$38-3067$ 


\section{ABSTRACT}

Cross-linked samples of natural rubber (NR) and synthetic cis-1,4-polyisoprene (IR) were instantaneously expanded to a predetermined strain ratio, $\alpha_{s}$, using a newly-designed high-speed tensile tester. Crystallization behavior after the cessation of deformation was investigated. The high-cycle wide angle X-ray diffraction (WAXD) measurements could successfully reveal the drastic progress of crystallization within the first a few hundred milliseconds. Quantitative analysis of diffraction intensity clarified coexistence of fast and slow crystallization processes; time constants $\tau_{f}$ and $\tau_{s}$, and amplitude $I_{f}$ and $I_{s}$, respectively, were estimated for these processes. The values of $\tau_{f}$ were in the range of 50 to $200 \mathrm{~ms}$, while $\tau_{s}$ ranged between 2.5 and $4.5 \mathrm{~s}$. Almost linear dependence of $I_{f}$ and $I_{s}$ on $\alpha_{s}$ was clarified. The crystallite size in the directions both parallel and perpendicular to the stretching direction decreased with the increase in timeaveraged nominal stress. The crystal lattice deformed almost linearly with the average nominal stress. For the fast process, correlation between crystallization and stress relaxation was not recognized, while linear relationship between them was found for the slow process. In every case, strain-induced crystallization was found to be the major origin of stress relaxation. Based on the results, effects of strain on crystallization of polymer melt were discussed.

\section{Keywords}

strain-induced crystallization, stress relaxation, injection molding, cross-linking 


\section{Introduction}

After the discovery of crystallization of conventional polymers into thin lamellae [1-4], this feature was found to be general among crystalline linear flexible chain polymers. In order to explain this peculiarity, the kinetic theories [5-8] based on the secondary nucleation were proposed. They could successfully explain the temperature dependences of the growth rate and the lamellar thickness. On the basis of these theories, we can now predict, to some extent, crystallization behavior of variety of polymer species. It is noted that these theories focus on the crystal growth in the quiescent polymer melt.

Many of polymer products are manufactured by the injection molding where high degree of strain is applied to the material. In the case of strained polymer melt, however, we have little knowledge about crystallization, especially in terms of kinetics. This is due to experimental difficulties on quantification of extremely fast nucleation and growth in strained and supercooled melt. Because the properties of polymer products are largely affected by their internal fine structures, quantitative guides about crystallization in strained polymer melt are desired.

Recently, the step shear technique is going to be used to study shear-induced crystallization quantitatively [9-13]. This technique is suitable to investigate the formation of oriented precursors of crystallization. However, the matrix polymer is quickly relaxed after cessation of the step shear and the strain is virtually removed through the process of disentanglement of molecular chains. For more detailed understanding of crystallization in strained polymer melt during the practical polymer processing, we need complementary knowledge about crystallization under a constantly-applied strain. Experiments for this kind of study require suppression of macroscopic relaxation; otherwise the effective amplitude of strain may change with the progress of relaxation. A way to suppress the macroscopic relaxation is the introduction of crosslinks into the sample, even though the complete suppression of relaxation is still difficult. That is to say, the studies on strain-induced crystallization of polymer network should contribute to broaden knowledge also about crystallization of strained polymer melt.

From the experimental viewpoint, when we investigate the crystallization behavior as a function of crystallization temperature, we have to change the temperature fast enough before the considerable progress of the structure formation. This requirement limits the observation temperature range when the crystallization rate of the sample is very fast. Thus, for example, the growth rates of polyethylene have not been measured at large supercooling, namely at low 
temperature. The same situation occurs when we study the strain-induced crystallization of polymer network. In this case, the driving force of crystallization is attributed to the reduced melting temperature due to the forced orientation of molecular chains in the stretching direction. Here, we consider the entropy change of deformation from $\alpha=1$ to $\alpha=\alpha_{1}$, namely $\Delta S_{d e f}$. (In this article, strain ratio $\alpha$ is defined as the ratio of the length of stretched sample to the unstretched one.) Melting temperature, $T_{m}\left(\alpha_{1}\right)$, of polymer network that is stretched to $\alpha_{1}$ of strain ratio is written as $[14,15]$;

$\frac{1}{T_{m}\left(\alpha_{1}\right)}=\frac{1}{T_{m}(1)}+\frac{\Delta S_{d e f}}{\Delta H(1)}$,

where $T_{m}(1)$ and $\Delta H(1)$ are melting temperature and the heat of fusion, respectively, of unstretched sample. When $T_{m}\left(\alpha_{1}\right)$ exceeds the ambient temperature and attains sufficient supercooling, strain-induced crystallization can take place. Analytical formulation of $\Delta S_{d e f}$, and hence the explicit form of Eq. 1, depends on the description of the strain-energy function. For example, when the simplest expression of $\Delta S_{d e f}$ assuming the Gaussian chains is applied [16], Eq. 1 is rewritten as;

$\frac{1}{T_{m}\left(\alpha_{1}\right)}=\frac{1}{T_{m}(1)}-\frac{v k\left(\alpha_{1}^{2}+2 / \alpha_{1}-3\right)}{2 \Delta H(1)}$,

where $v$ is network-chain density and $k$ is the Boltzmann constant. By the more rigorous treatment, Flory proposed the next formula [17],

$\frac{1}{T_{m}\left(\alpha_{1}\right)}=\frac{1}{T_{m}(1)}-\frac{R}{\Delta H(1)}\left[\left(\frac{6 m_{0} v}{\pi \rho}\right)^{1 / 2} \alpha_{1}-\frac{m_{0} v}{\rho}\left(\frac{\alpha_{1}^{2}}{2}+\frac{1}{\alpha_{1}}\right)\right]$

where $R$ is the gas constant, $m_{0}$ the mass of statistical segments and $\rho$ the specific gravity. Regardless to the explicit form of Eq. 1, supercooling increases with the increase in strain ratio. (Note that experimental results do not necessarily follow the predictions from Eqs. $1 \sim 3[15,18$ 21]. These formulas were presented here only for understanding of the basic idea.) At the same 
time, the time for conformation change to fit into the crystal lattice is drastically reduced in the stretched sample. As a result, the rate of strain-induced crystallization is accelerated with the increase in strain ratio, $\alpha_{1}$. For the investigation of strain-induced crystallization, therefore, we have to expand the sample fast enough before the considerable progress of crystallization, especially when the sample is highly strained.

Experiments belonging to this category have been carried out since 1930s (before the discovery of the lamellar crystals [1-4]). Relatively fast crystallization behavior of cross-linked natural rubber (NR) at high degree of fixed strain $\left(\alpha_{s}\right)$ has been investigated by Acken et al [22] and Long et al. [23] using a special instrument designed for this purpose. They have reported about the existence of induction period before the onset of crystallization after high degree of strain was applied. Later study by Dunning \& Pennells [24] using a different type of instrument also reported about the induction period which varies from $50 \mathrm{~ms}$ to $18 \mathrm{~s}$ according to $\alpha_{s}$. In these studies, however, kinetics of strain-induced crystallization was merely discussed. The time constant of relatively fast strain-induced crystallization was first estimated to be tens of milliseconds by Mitchell \& Meier [25] on the basis of thermal and tensile measurements using a high-speed tensile tester. (There were other studies on relatively slow kinetics of strain-induced crystallization for moderately strained samples of cross-linked NR [26-29]. According to these studies, two or more types of crystallization processes may coexist in the time scale ranging between a few minutes and several months. Such slow processes are qualitatively different from the ones in the current study. In the latter part of this article, for simplicity, the terms "fast" and "slow" mostly refer to the phenomena in the time scale ranging between tens of milliseconds to ten seconds.)

We also have studied the crystallization behavior of highly stretched samples of NR and synthetic cis-1,4-polyisoprene (IR) [30,31]. On the basis of the development of wide-angle X-ray diffraction (WAXD) intensity with elapsed time, time constants of strain-induced crystallization at $\alpha_{s}=6$ were estimated to be $2 \sim 5$ seconds. In these studies, however, crystallization has considerably progressed during the deformation process due to the very fast rate of crystallization, though the maximum speed of the tensile tester was used. (This tensile tester has not been designed for the fast deformation experiments.) Therefore, we could not mention about very early stage of crystallization; also the range of strain ratio was limited. The time constants that we obtained were different from the ones reported by Mitchell \& Meier [25]. It remained vague why the different time constants by two orders of magnitude have reported by these studies. 
The above-mentioned background has motivated us to carry out high-cycle time-resolved WAXD measurements in combination with a high-speed tensile tester to clarify the initial behavior of strain-induced crystallization in polymer network, and hence, of oriented and supercooled polymer melt. In this study, we newly devised the high-speed tensile tester and put the high-cycle WAXD experiments into practice using a powerful synchrotron light source. Crystallization from highly-strained polymer melt is discussed on the basis of the results of this study.

\section{Experimental}

\subsection{Materials}

Sheets (1 mm thick) of vulcanized NR and IR samples with different network-chain densities were kindly supplied from Toyo Tire and Rubber Co., Ltd. The recipes for the preparation of the samples are listed in Table 1 [18,19]. Values of network-chain density, $v$, were estimated from the tensile properties on the basis of the rubber elasticity theory [16] and are listed in Table 2 along with the curing time. The cure temperature was $140{ }^{\circ} \mathrm{C}$ for all the samples.

Ring-shaped specimens were die-cut from the sample sheets. The width and circumference of the specimens were ca. $1 \mathrm{~mm}$ and $50 \mathrm{~mm}$, respectively. The initial length corresponds to the half of the circumference (i.e., $25 \mathrm{~mm}$ ).

Table 1 Recipes of vulcanized rubber samples in part.

\begin{tabular}{lllllll}
\hline Sample & Rubber & Stearic acid & $\mathrm{ZnO}$ & $\mathrm{CBS}^{\mathrm{c}}$ & Sulfur & Total \\
\hline NR1T & $100^{\mathrm{a}}$ & 2 & 1 & 3 & 4.5 & 110.5 \\
NR3T & $100^{\mathrm{a}}$ & 2 & 1 & 1.5 & 2.25 & 106.75 \\
NR5T & $100^{\mathrm{a}}$ & 2 & 1 & 0.75 & 1.125 & 104.88 \\
IR3T & $100^{\mathrm{b}}$ & 2 & 1 & 1.5 & 2.25 & 106.75 \\
IR5T & $100^{\mathrm{b}}$ & 2 & 1 & 0.75 & 1.125 & 104.88 \\
\hline
\end{tabular}

${ }^{\mathrm{a}} \mathrm{RSS}$ \#3

${ }^{\mathrm{b}} \operatorname{IR} 2200$ 
${ }^{\mathrm{c}} N$-cyclohexyl-2-benzothiazole sulfenamide

Table 2 Curing time and network-chain density of vulcanized rubber samples

\begin{tabular}{lll}
\hline Sample & Curing time $(\mathrm{min})$ & $v \times 10^{4}\left(\mathrm{~mol} / \mathrm{cm}^{3}\right)$ \\
\hline NR1T & 20 & 2.44 \\
NR3T & 25 & 1.95 \\
NR5T & 35 & 1.48 \\
IR3T & 35 & 1.76 \\
IR5T & 50 & 1.49 \\
\hline
\end{tabular}

\subsection{WAXD experiments}

As was so in our previous experiments [30,31], the specimen was expanded to a predetermined strain ratio, $\alpha_{s}$, and the deformation was immediately stopped. On the basis of the results in our previous study [18], the range of $\alpha_{s}$ was determined to be $4-7$. Tensile stress and twodimensional WAXD patterns were recorded simultaneously during and after deformation. In this paper, we consistently describe elapsed time, $t$, in unit of second and define the origin of the elapsed time $(t=0 \mathrm{~s})$ at the cessation of the deformation. The experiments were performed at BL40XU beam line in SPring-8, Japan, where high-flux monochromatic X-ray beam for the fast time-resolved experiments is available. The ambient temperature was controlled at $25{ }^{\circ} \mathrm{C}$. The wave length was $0.0832 \mathrm{~nm}(15 \mathrm{keV})$ and the camera length was ca. $180 \mathrm{~mm}$. The drawing axis of the specimen was titled to adjust the 002 reflection to satisfy the Bragg reflection condition. In this study, the shorter cycle of time-resolved WAXD measurements and the faster rates of deformation of the specimen compared to the previous studies were targeted. The exposure condition was adjusted more carefully, and as a result, the two-dimensional WAXD patterns were recorded every 36 ms using a Hamamatsu C4880-80 CCD camera.

In order to accomplish the fast deformation, a custom-made tensile tester was placed on the beam line. This tensile tester equipped a specially-designed specimen chuck in a similar way as in ref. [32], which enabled WAXD analysis of a fixed part of the specimen. The maximum deformation rate of the instrument was $1,000 \mathrm{~mm} / \mathrm{s}(60,000 \mathrm{~mm} / \mathrm{min})$, which was faster than the 
previous experiments $[30,31]$ by two orders. By using this high-speed tensile tester, the deformation of the specimen could be completed instantaneously. The high-speed deformation, however, accompanied a problem of specimen vibration after the cessation of deformation, which affected both the tensile and the WAXD measurements. In order to minimize this problem, the maximum deformation rate $(1,000 \mathrm{~mm} / \mathrm{s})$ was applied only for the case of $\alpha_{s}=7$; for the other cases $\left(\alpha_{s}=4,5\right.$ and 6$)$, the deformation rate was reduced to $250 \mathrm{~mm} / \mathrm{s}$. That is, the specimen was deformed to the prefixed $\alpha_{s}$ at $1,000 \mathrm{~mm} / \mathrm{s}\left(40 \mathrm{~s}^{-1}\right)$ or $250 \mathrm{~mm} / \mathrm{s}\left(10 \mathrm{~s}^{-1}\right)$ and allowed to relax for $10 \mathrm{~s}$. No essential difference due to the difference in deformation rate was found in our analysis.

\subsection{Processing of the WAXD data.}

The data were processed almost similarly to our previous works [30,31]. Rectangular regions including 200, 120 and 002 reflections were selected (indicated by solid lines in Fig. 1). First, intensity was integrated over each of the rectangular region, and then normalized using integrated intensity of rectangular regions on the both sides. The intensity value ( $I_{h k l}(t)$ for the $h k l$ reflection) was estimated for each of the selected reflection [30]. In order to monitor the development of crystallinity, the values of $I_{200}(t), I_{120}(t)$ and $I_{002}(t)$ were averaged. Thus estimated average intensity is denoted as $I(t)$. Next, the profile of the reflection peak was analyzed [31]. The intensity of pixels in each selected region were summed up in the direction parallel to the meridian (for 200 and 120 reflections) or to the equator (for the 002 reflection). In this way, we obtained the quasi radial intensity profile for each of the crystalline reflection. After subtraction of the linear base line, each diffraction profile was fitted with the Gaussian function;

$$
G(x)=h \cdot \exp \left[-\left(x-x_{\mathrm{c}}\right)^{2} /\left(2 \cdot w^{2}\right)\right]
$$

where $x$ is the coordinate in pixel unit, $x_{c}$ is the position of the peak, $h$ and $w$ correspond to the height and width of the reflection, respectively. The values of $x_{c}, h$ and $w$ were estimated as the fitting parameters. Each of the $w$ value was converted into the half-width $\beta$ without the deconvolution of the instrumental broadening [19]. Then the crystallite size was estimated by using the Scherrer equation; 


$$
L_{h k l}=K \cdot \lambda /(\beta \cdot \cos \theta)
$$

where $L_{h k l}$ is the crystallite size in the direction perpendicular to the $(h k l)$ plane, $\lambda$ is the wavelength and $\theta$ is the Bragg angle (half of the scattering angle). The value 0.89 was used for $K$ [33]. Finally, the dimensions of the crystal unit cell $(a, b$ and $c$ ) were estimated for the strained specimens on the basis of the peak position, $x_{\mathrm{c}}$. The above-mentioned procedures were accomplished for thousands of WAXD data files by using homemade software. Note that the absolute values of the crystallite size and the dimensions of the unit cell involve certain degree of deviations. For example, the distortion of the WAXD pattern projected on the spherical screen of the CCD camera was not corrected, which should affect the unit cell dimensions. Because the instrumental broadening was not deconvoluted, the crystallite size should be estimated to be smaller than the real value. When the crystal reflections were weak, fitting error was relatively large. By considering these inaccuracies, we will limit the discussion on the relative changes and the differences among the samples for these values.
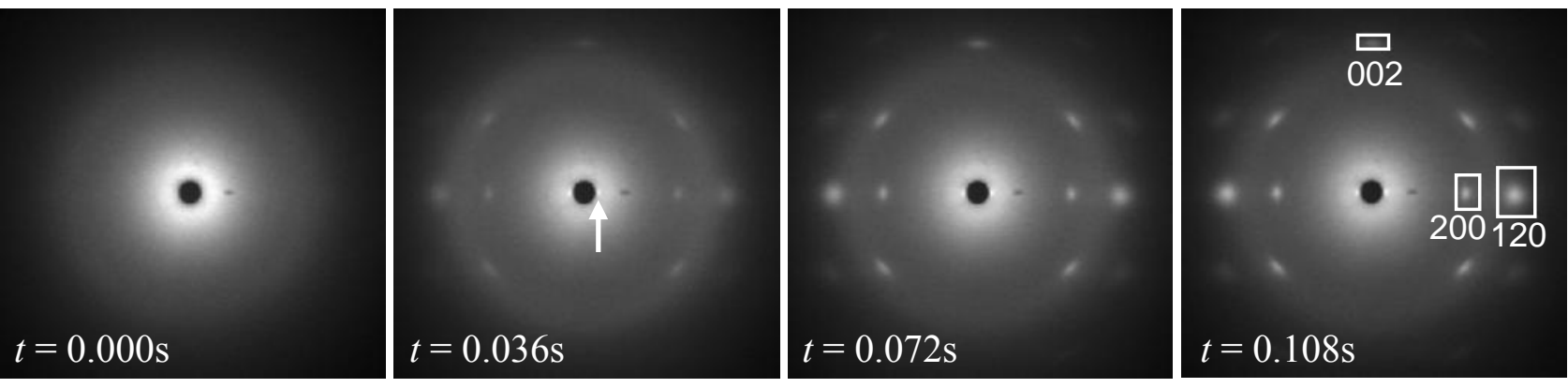

Fig.1

\section{Results}

\subsection{Crystallization processes}

Crystallization of NR samples was confirmed for all the $\alpha_{s}$ values $\left(\alpha_{s}=4 \sim 7\right)$ except NR1T which was broken during the deformation to $\alpha_{s}=7$. On the other hand, IR samples did not crystallize in this experiment when $\alpha_{s}=4$, as expected from the result of the cyclic deformation [18]. Because the drawing axis of the specimen was tilted to adjust one 002 reflection to satisfy 
the Bragg reflection condition, the counterpart ( $00 \overline{2}$ reflection) was not observed. It was our primary concern whether we could record the very early stage of strain-induced crystallization using the high-speed tensile tester. Fig. 1 shows examples of the WAXD patterns within ca. $0.1 \mathrm{~s}$ of elapsed time. As shown in this figure, when the deformation was completed (at $t=0.000 \mathrm{~s}$ ), no or very weak crystalline reflections were recognized. This result indicates that we could successfully obtain the WAXD patterns from the onset of strain-induced crystallization. It is surprising to find that the WAXD pattern at $t=0.000 \mathrm{~s}$ is fairly isotropic though the specimen is highly stretched $\left(\alpha_{s}=7\right)$. Furthermore, reflections from stearic acid crystals which should be located near the beam stop are not recognized in this initial WAXD pattern. In the subsequent WAXD patterns, sharp reflections from stearic acid (indicated by the arrow) appeared, and furthermore, the amorphous halo increased the intensity on the equator. These features were observed for several cases, suggesting reorganization of the network structure within a few tens of milliseconds after the cessation of deformation.

Strain-induced crystallization then progressed drastically within a few hundred milliseconds. In order to analyze the crystallization kinetics quantitatively, intensity values of the crystalline reflections were estimated. Fig. 2a shows an example of the development of $I_{200}(t), I_{120}(t)$ and $I_{002}(t)$ with elapsed time. Every reflection shows the similar dependence on the logarithm of elapsed time, $t$. In Fig. 2a, just after the cessation of the deformation, $I_{h k l}(t)$ values increase rapidly, showing the steep slope in the plot, and then the slope changes to be moderate. This feature implies that there are two crystallization processes with different time constants. The same feature was observed for all the cases in the current study when strain-induced crystallization was confirmed.

According to our preliminary analysis, no essential difference was found about the behavior of $I_{h k l}(t)$ regardless to indices of the selected reflections. Therefore, we calculated the average of the $I_{h k l}(t)$ values, namely $I(t)$, to reduce the experimental fluctuations for further analysis. Examples of the development of $I(t)$ with elapsed time are shown in Fig. $2 \mathrm{~b}$. Then $I(t)$ was fitted using the formula;

$I(t)=I_{0}+I_{f}\left[1-\exp \left(-t / \tau_{f}\right)\right]+I_{s}\left[1-\exp \left(-t / \tau_{s}\right)\right]$ 
where $\tau_{f}$ and $\tau_{s}$ are the time constants of the crystallization processes $\left(\tau_{f}<\tau_{s}\right) ; I_{f}$ and $I_{s}$ are, respectively, the amplitude of these processes and $I_{0}$ is a constant related to the initial value. The resultant fitting curves are also indicated in Fig. 2. The fitting parameters $\tau_{f}, \tau_{s}, I_{f}$ and $I_{s}$ are plotted in Figs. 3 and 4 as a function of $\alpha_{s}$. The values of $\tau_{f}$ were in the range of 50 to $200 \mathrm{~ms}$ (Fig. 3a), which were similar to the time constants reported by Mitchell \& Meier [25]. On the other hand, $\tau_{s}$ in this study corresponds to the time constants reported in our previous study [30]. It has been a question why the different time constants were obtained in these two previous studies, and now we confirmed that the two types of crystallization processes with different time constants do coexisted. The experimental method by Mitchell \& Meier was insensitive to slow crystallization processes due to heat dissipation, and accordingly, the process having the longer time constants might not be detected. On the other hand, the fast process was detectable only with the specially designed high-speed tensile testers. It is emphasized that this should be the first report that clarified the coexistence of the fast and the slow processes during the early stage of strain-induced crystallization in highly stretched polymer networks.

According to Fig. 3, the time constants do not show definite dependence on the strain ratio, $\alpha_{s}$. On the other hand, almost linear dependence of $I_{f}$ and $I_{s}$ on $\alpha_{s}$ is clearly perceived in Fig. 4.The amplitude $I_{f}$ and $I_{s}$ are related to the amount of molecular chains that can crystallize during the corresponding processes. It is interesting to find that network-chain density did not affect $I_{f}$ and $I_{S}$ that much, considering the theoretical expectation that the supercooling may depend on network-chain density. On the other hand, the difference between NR and IR samples shifted the entire plots. The latter feature is attributable to the lower melting temperature of IR [34,35] due to the lower regularity in the main-chain structure [36,37]. 

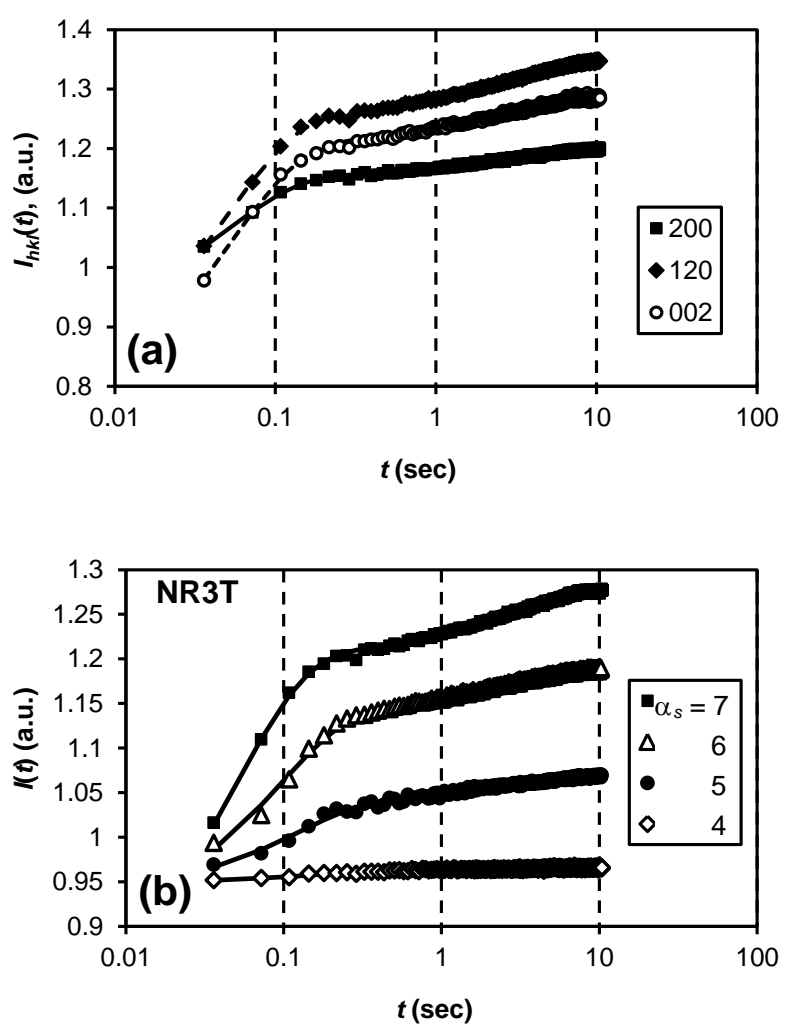

Fig. 2
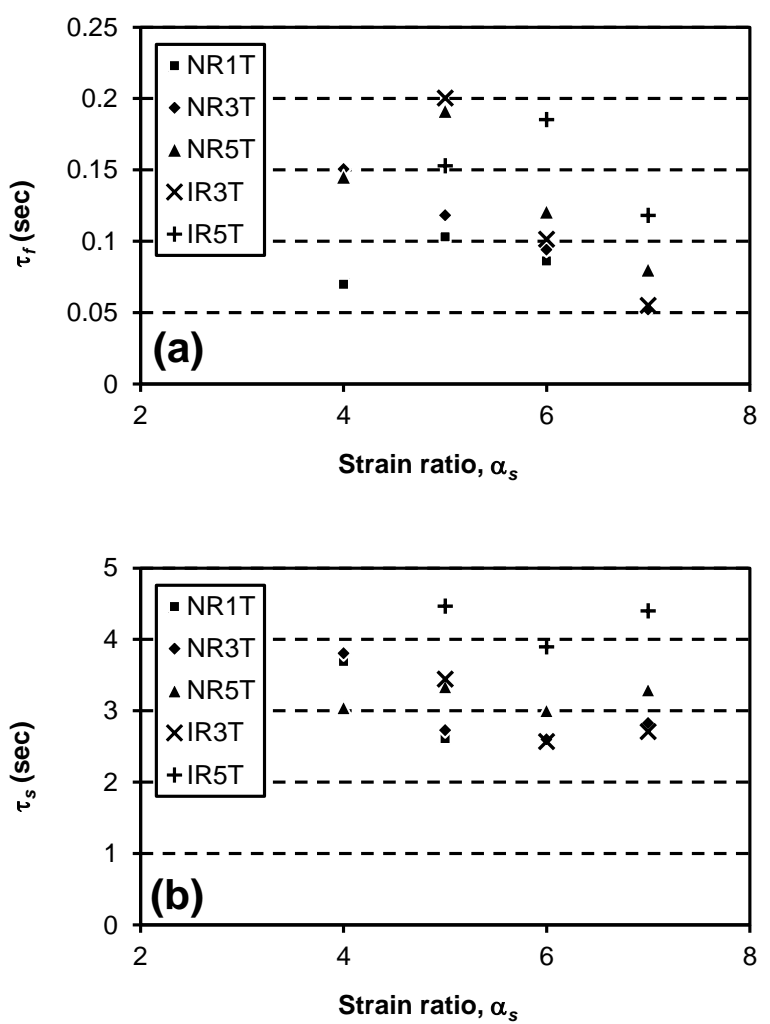

Fig. 3 

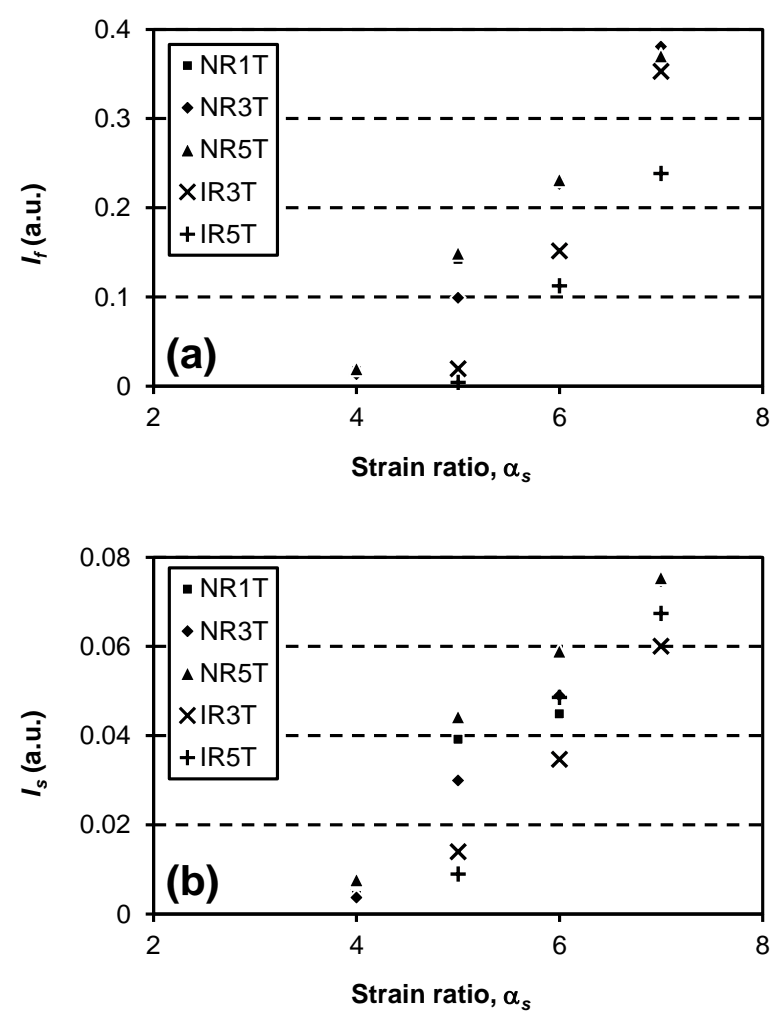

Fig. 4

\subsection{Morphological features}

Morphological changes during the early stage of crystallization under constant strain were drawn out from the WAXD patterns. Fig. 5 shows examples of the dependence of crystallite size, $L_{200}$, on elapsed time, $t$. Though this figure shows relatively small scatter, the estimated values fluctuated much in some other cases. Especially, when the reflections were weak (at short $t$ and for small $\alpha_{s}$ ), the range of fluctuation was large (not only for the crystallite size but also for the dimensions of crystal unit cell). Nevertheless, we tried to read out general trends and judged that the crystallite size was almost constant during the observed period of elapsed time, as shown in this figure. We therefore compared the time-averaged crystallite size, $\left\langle L_{h k l}>\right.$. The crystallite size decreased linearly with the increase in network-chain density (v), and at the same time, with the increase in $\alpha_{s}$. Both of the increases in $v$ and $\alpha_{s}$ result in the higher tensile stress. Accordingly, the dependence of crystallite size on the time-averaged value of nominal stress was examined. Figs. $6 \mathrm{a}$ and $6 \mathrm{~b}$ show the dependence of $\left\langle L_{200}>\right.$ and $\left\langle L_{002}>\right.$, respectively, on the average nominal stress. Because120 reflection includes effect of the statistical displacement of molecular chains in 
the crystal structure of NR [38,39], $L_{120}$ was excluded from the analysis. (According to the crystal structure analysis by Nyburg [38] and by Takahashi \& Kumano [39], the molecular chains in the unit cell are statistically replaced with those related by the mirror symmetry at $y=1 / 8$. By this replacement, $L_{120}$ is reduced, while projection of the molecular chains on the ac-plane, and hence $L_{200}$, do not change.) In Fig. 6c, the crystallite size is plotted as a function of inverse of the average nominal stress, showing the good linear relationship regardless to the difference in samples. In this way, the crystallite size decreased with the increase in average nominal stress in the directions both parallel and perpendicular to the stretching direction, though it was insensitive to the stress relaxation with elapsed time.

The similar results are obtained for the dimensions of the crystal unit cell. Though the data fluctuated considerably in some cases, the dimensions of unit cell seemed to be almost constant in the current experiment. Therefore, the average values of the unit cell were investigated. As shown in Fig. 7, the dimensions of unit cell changed almost linearly with the average nominal stress, that is to say, the crystal lattice distorted due to the applied stress. We have already detected the distortion of crystal lattice during the continuous deformation of the NR samples [19]. The results of the current study suggested that the lattice distortion occurred at the onset of crystallization and remained during the subsequent crystallization process.

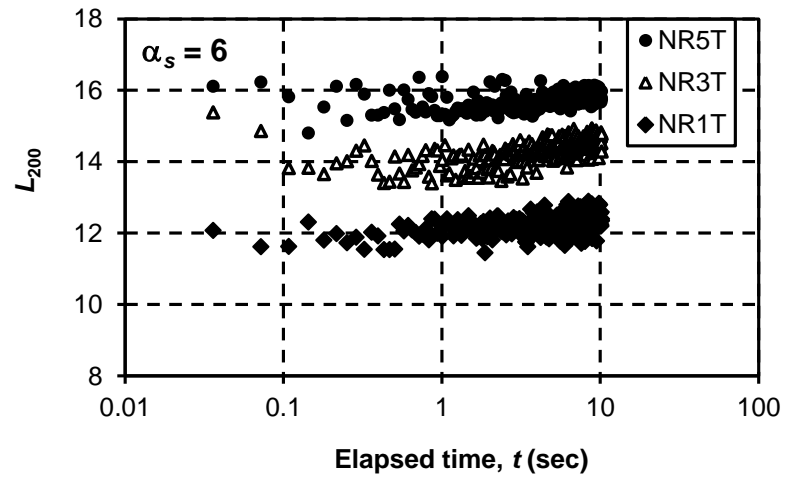

Fig. 5 

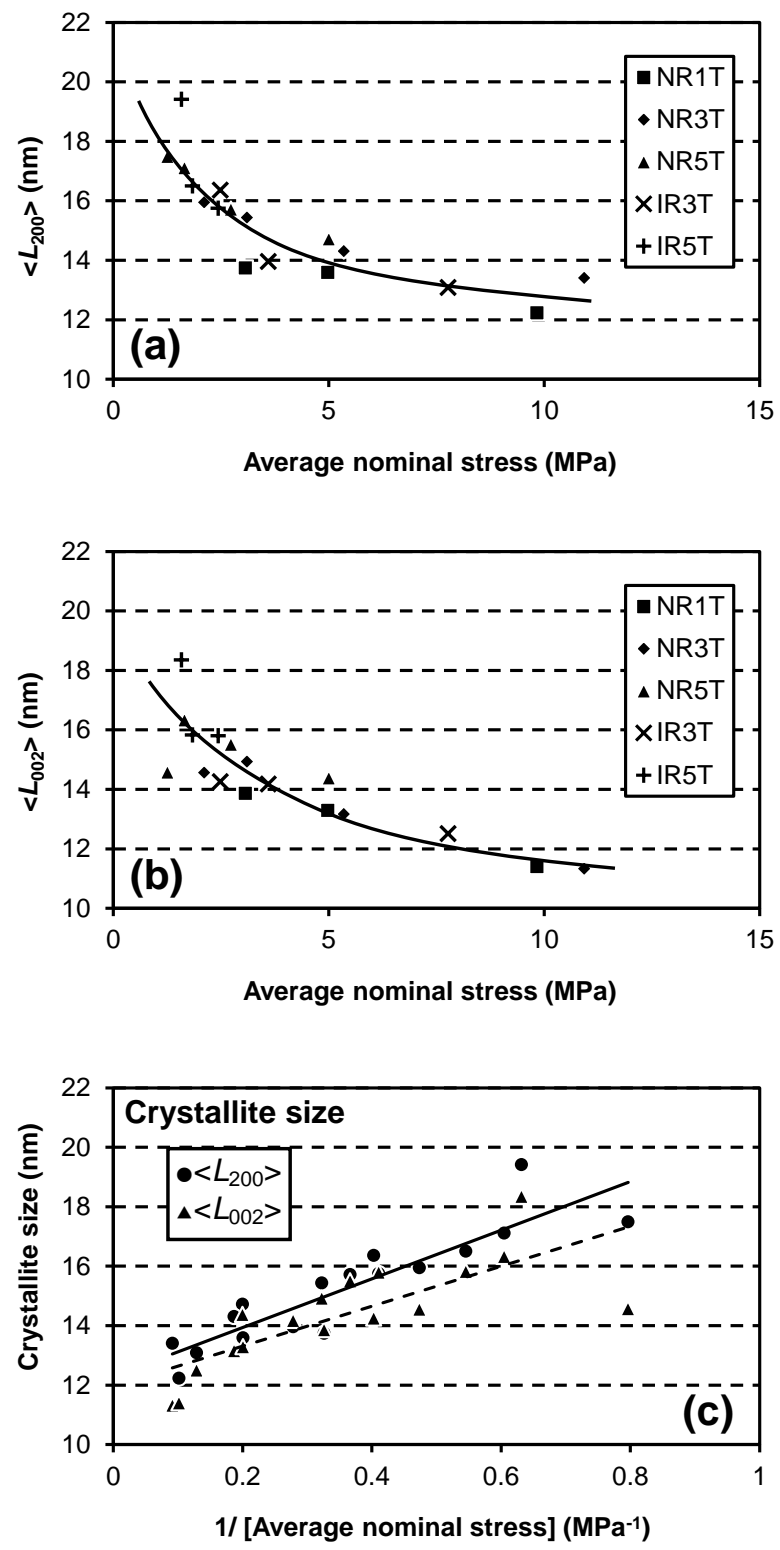

Fig. 6 

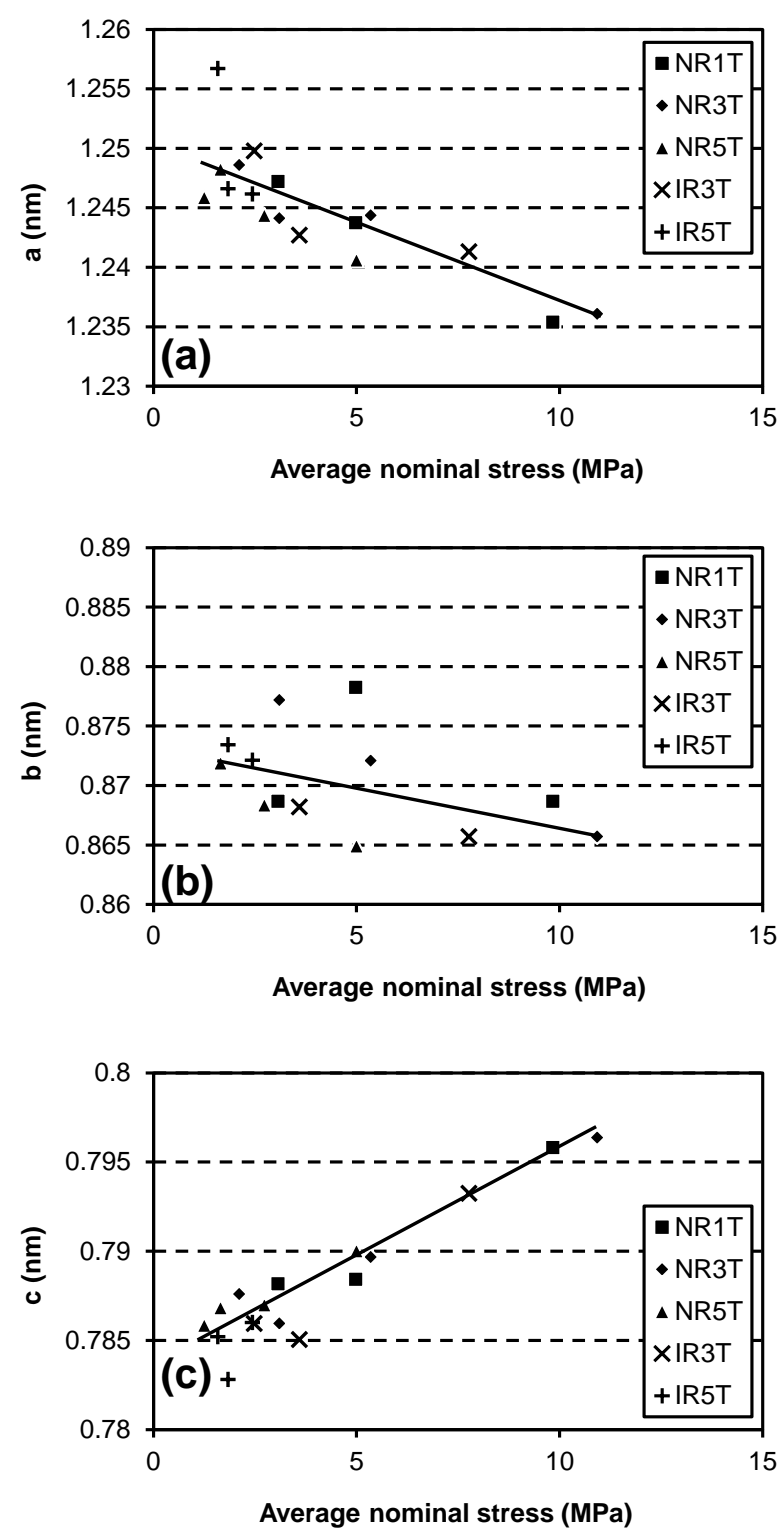

Fig. 7

\subsection{Stress relaxation}

The occurrence of stress relaxation due to strain-induced crystallization has been theoretically predicted $[17,40]$ and experimentally confirmed by many researchers $[19,26,34,35,41-45]$. As a result of crystallization, parts of molecular chains are stabilized in the extended conformation aligned in the stretching direction. The rest parts of the chains in the amorphous phase can adopt more relaxed conformation, exhibiting less stress that originates from the entropy elasticity. In 
our previous work [30], correlation between the amplitude of crystallization and stress relaxation has already been reported. The results indicated that strain-induced crystallization is the major origin of the stress relaxation. However, only the NR samples could be analyzed and the condition was limited to $\alpha_{s}=6$ due to the considerable progress of crystallization during deformation. In the current study, thanks to the instantaneous deformation, wider range of $\alpha_{s}$ values and two types of crystallization processes were accessible for both the NR and IR samples. We have therefore applied the similar method to the analysis of stress relaxation for variety of $\alpha_{s}$ values, considering the two crystallization processes. Because the initial values of tensile stress (at $t=0 \mathrm{~s}$ ) changed according to the network-chain densities and to $\alpha_{s}$ values, the degree of stress relaxation was compared after normalization of measured stress using the maximum stress value at $t=0$. The stress value after the normalization is denoted as $\sigma(t)$. Fig. 8 shows the examples of stress relaxation behaviors of NR3T at various $\alpha_{s}$. Then the stress relaxation behavior was fitted with the next formula;

$$
\sigma(t)=\sigma_{0}-\sigma_{f}\left[1-\exp \left(-t / \tau_{f}\right)\right]-\sigma_{s}\left[1-\exp \left(-t / \tau_{s}\right)\right]-\sigma_{x}\left[1-\exp \left(-t / \tau_{x}\right)\right]
$$

where $\sigma_{0}$ is the initial value of the normalized stress $(\simeq 1), \sigma_{f}, \sigma_{s}$ and $\sigma_{x}$ are the relative amplitude of stress relaxation having the corresponding time constant. The second and third terms of Eq. 7 represent contributions of strain-induced crystallization; their time constants $\tau_{f}$ and $\tau_{s}$ have been estimated beforehand from the WAXD data (Fig. 3). The fourth term having the time constant $\tau_{x}$ was introduced to consider other relaxation processes (e.g., effect of chain disentanglement). The obtained fitting parameters are plotted in Figs. 9 and 10. The values of $\tau_{x}$ (Fig. 9) are close to the corresponding ones in our previous report ( $\tau_{2}$ in ref. [30]) and were between $\tau_{f}$ and $\tau_{s}$. Finally, the correlation between the amplitude of crystallization $\left(I_{f}\right.$ and $I_{s}$ in Fig. 4) and stress relaxation $\left(\sigma_{f}\right.$ and $\sigma_{s}$ in Fig. 10) was examined. For the fast process, correlation between $I_{\mathrm{f}}$ and $\sigma_{f}$ was not recognized (Fig. 11a). On the other hand, linear relationship between $I_{S}$ and $\sigma_{s}$ was found for the slow crystallization process, as shown in Fig. 11b. (In this figure, three points which are enclosed by the broken line deviate from the linearity. These exceptions correspond to $\alpha_{s}=7$. It is assumed that the limited mobility of network chains at this high strain ratio has led to this deviation.) During the fast process, despite the considerable progress of 
crystallization, the amplitude of stress relaxation was small. Accordingly, the ratio, $\sigma_{f} / I_{f}$ was by one or more orders smaller than $\sigma_{s} / I_{s}$ (Fig. 12). It is interesting to find that $\sigma_{s} / I_{s}$ increases with the decrease in $\alpha_{s}$. In this way, the fast and the slow processes of strain-induced crystallization were found to be different with each other. While stress relaxation progressed with the progress of the slow crystallization process, merely trivial relaxation occurred during the fast process. It is noted that, even considering the existence of the fast process, the major origin of stress relaxation was still the strain-induced crystallization. This is indicated in Fig. 13, in which the relative contribution of strain-induced crystallization to the total degree of stress relaxation, $\left(\sigma_{f}+\sigma_{s}\right) /\left(\sigma_{f}\right.$ $\left.+\sigma_{s}+\sigma_{x}\right)$, is plotted. All the values are larger than 0.5.

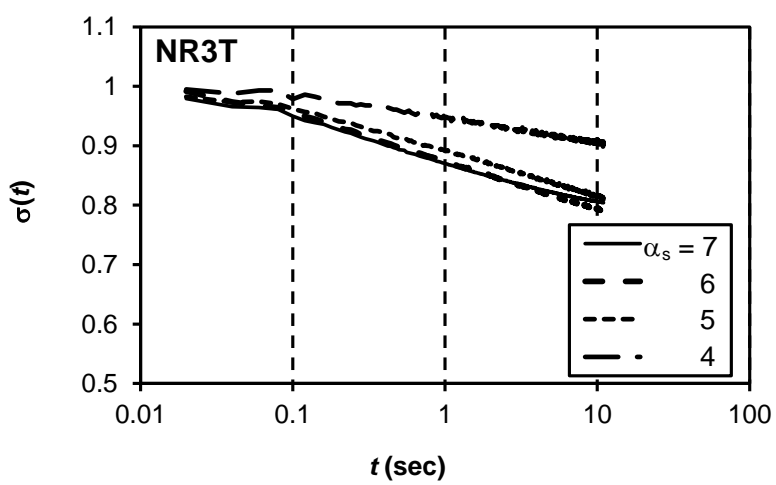

Fig. 8

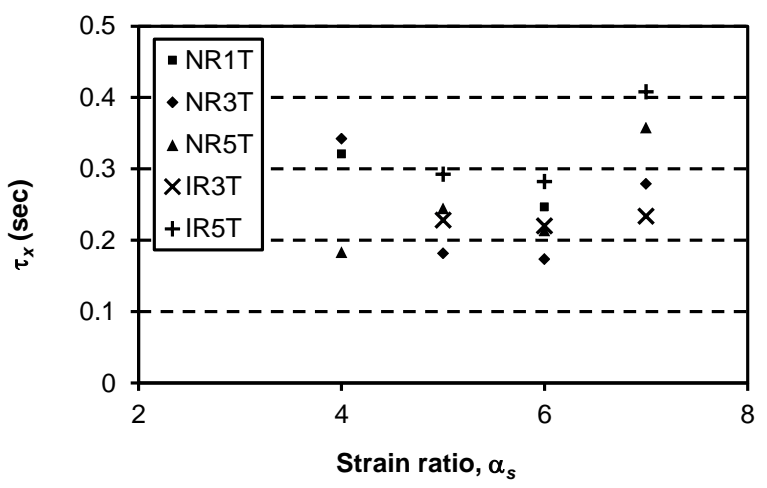

Fig. 9 

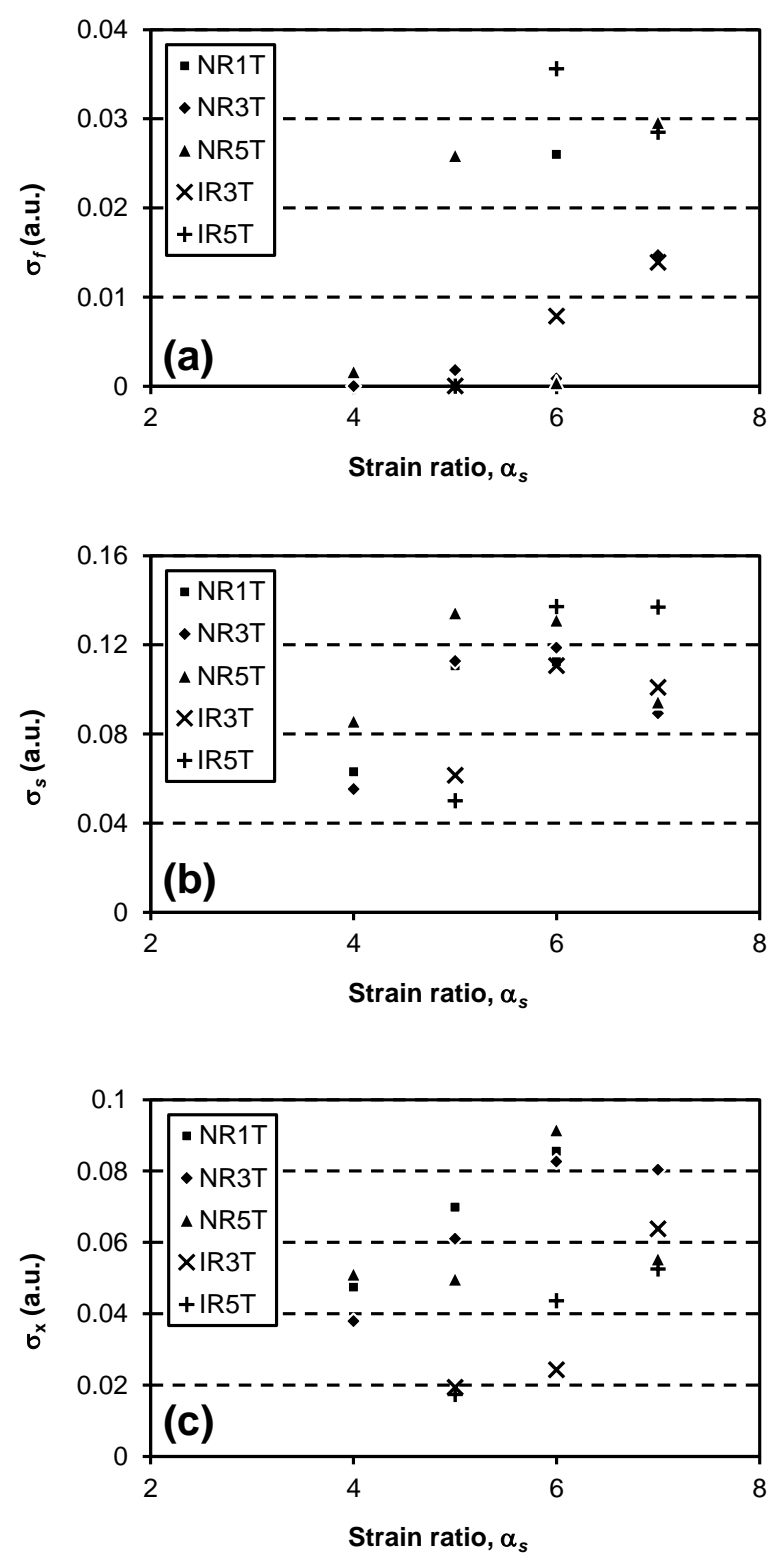

Fig. 10 

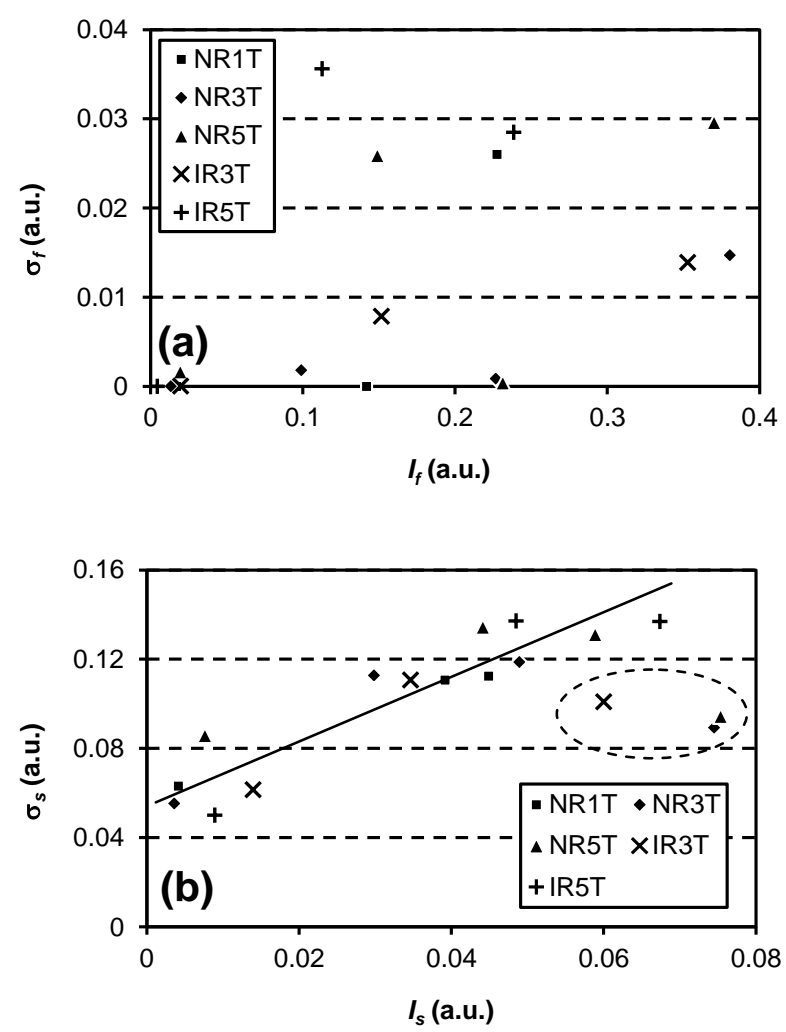

Fig. 11
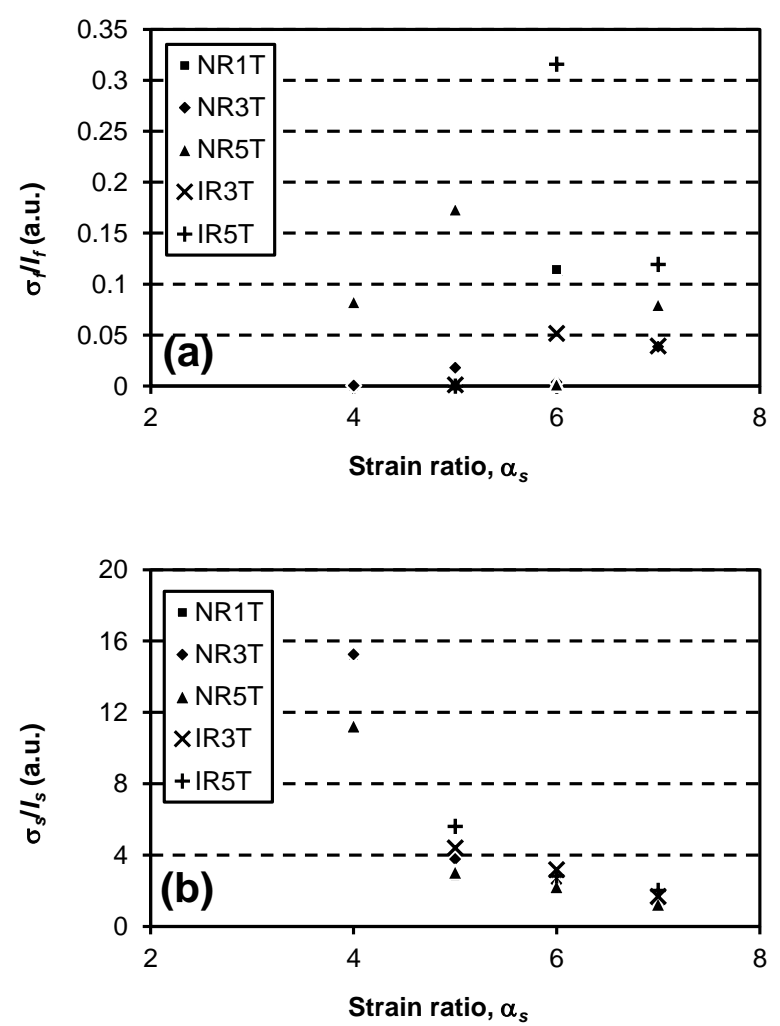

Fig. 12 


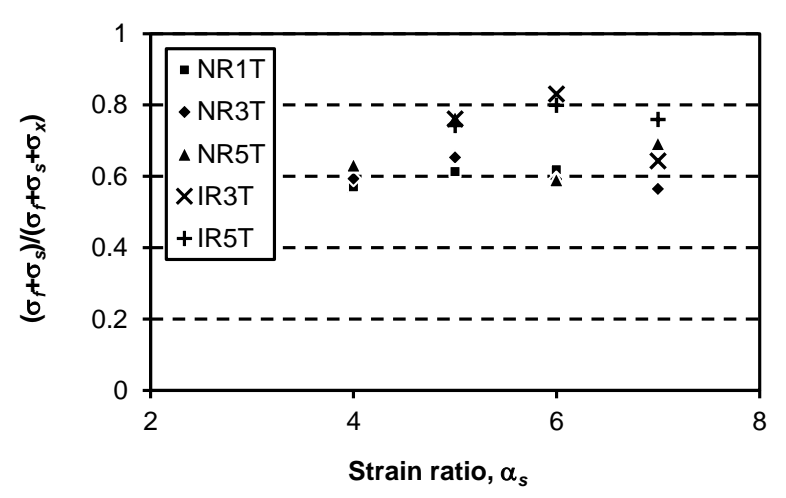

Fig. 13

\section{Discussion}

The finding of the coexistence of the fast and the slow processes of strain-induced crystallization has led us to the following two questions. What is the basic difference between the fast and the slow processes? Why the fast process does not accompany commensurate stress relaxation? For the moment, we have hypotheses to answer these questions, though they are not fully consistent with all the observed features in this and previous studies.

The fast process is thought to be the crystallization of highly-aligned molecular chains that require only limited time to fit into the crystal lattice via, e.g., small movement of local chain segments, while the slow process should include certain degree of diffusion of main chains to form the crystalline order. The amount of molecular chains that are related to each of the process should increase with the strain ratio, $\alpha_{s}$, which is consistent with the results in Fig. 4. In order to explain the smaller degree of stress relaxation during the fast process, the temperature rise is taken into account. As a result of rapid expansion and crystallization, the generated heat is accumulated in the specimen. In fact, Mitchell \& Meier [25] analyzed the fast crystallization process by the temperature measurements. Then the elevated temperature should increase the entropic elasticity, compensating the relaxation due to crystallization. The stress relaxation commensurate with the fast crystallization may come out later as the dissipation of the heat, partly contributing to the slower relaxation processes. Unfortunately, the above-mentioned hypotheses were not supported by the morphological features. If there are highly-aligned molecular chains before the onset of crystallization as supposed above, we can expect intensity localization of amorphous halo on the equator at $t=0.000 \mathrm{~s}$ (Fig. 1). The crystals generated from 
such molecular chains may have exhibited different degree of crystallite size and lattice distortion. The observed features were somewhat inconsistent with these expectations. Of course, the expected features could have existed, though they have been obscured by the detection limit.

We guess that some of the features found in the current study are applicable also to crystallization of other polymer species from strained melt. In the polymer melt, chain entanglements play the role of cross-linking points. Then the polymer melt is regarded as a kind of polymer network in the short period of time. When chain entanglements have sufficiently long life time, the fast and the slow processes of strain-induced crystallization could coexist, otherwise only the fast process may come out. This idea is convenient to understand the results of step shear experiments on isotactic polystyrene (i-PS) by Kanaya's group [11,13]. They observed formation of precursors of oriented crystals even above the nominal melting temperature of i-PS after the application of short-duration strain to the melt. (Recently, they confirmed that the precursors do include crystals on the basis of the micro-beam WAXD experiment [46].) The temperature range of the step-shear experiment was analogous to the current study that was performed at room temperature, i.e., above the nominal melting temperature of NR which is around $0{ }^{\circ} \mathrm{C}[34,47]$. Though the duration of step shear was short, the fast process might have the comparable time constant. Thus the formation of the precursors, namely the oriented crystalline nuclei, during the very short duration of strain can be explained by considering the fast process. In this way, the fast process is assumed to be related to the formation of oriented nuclei in strained polymer melt.

According to Fig. 4, the density of the crystallizable chains depends on the amplitude of strain but is insensitive to cross-linking density. In the case of uncross-linked polymer melt, accordingly, the density of oriented nuclei may also be insensitive to the density of entanglement. When the strain rate is large enough (higher than a critical value for disentanglement), the number of oriented nuclei is predicted to depend mainly on strain amplitude. This expectation is qualitatively consistent with the transmission electron microscopy observations by Andrews et al. $[48,49]$ and Shimizu et al. [50-52]. At room temperature, thin films of uncross-linked NR and chloroprene rubber were pre-stretched to predetermined strain. In this case, uncross-linked NR should also have the time constant $\left(\tau_{f}\right)$ of formation of the oriented nuclei around tens of milliseconds. Accordingly, the oriented nuclei commensurate with the degree of strain should have formed during the pre-stretching. As a result of the crystallization at low temperature, the 
number of oriented nuclei was indeed found to increase with the increase in strain ratio during the pre-stretch.

The more important implication of the results in this study lies in the consideration of the origin of acceleration of crystallization in strained polymer melt. As has been mentioned in introduction, the acceleration of crystallization is explained by the increased supercooling and the reduced time for conformational change to fit into the crystal lattice due to the orientation of molecular chains. Then one might have imagined that the time constants of nucleation and crystal growth are reduced from the ones in quiescent melt. The results in Figs. 3 and 4 suggest, however, that the acceleration is brought about by manifestation of the crystallization processes having the shorter but almost fixed time constants; with the increase in strain ratio, the more acceleration is derived because the fraction of molecular chains that can crystallize during the faster process increases.

Finally, we should comment on puzzling morphological features found in the studies on straininduced crystallization of cross-linked NR. The decrease of crystallite size in the stretching direction, namely $L_{002}$, with the increase in nominal stress is very difficult to comprehend (Fig. 2b). This feature has also been reported by Trabelsi et al. [53] during the cyclic deformation, and accordingly, is thought to be a general trend. Birefringence measurements suggested that highly oriented amorphous chains may have converted to oriented crystals in samples exhibiting high tensile stress [54]. Such oriented chains should show the localization of halo intensity on the equator. However, we have not seen such an indication in the WAXD patterns (Fig. 1, $t=0.000 \mathrm{~s}$ ). On the contrary, when the network-chain density is low and $\alpha_{s}$ is small, strain-induced crystals are thought to incorporate unoriented chains from the surrounding amorphous phase according to the large increase in birefringence [54]. In the WAXD patterns, the oriented crystalline reflections appear in the almost isotropic amorphous halo, as shown in Fig. 1. These observations suggest the conversion of coiled chains into aligned crystalline chain stems. Such a process is thought to accompany chain folding. However, no trace of formation of stacked-lamellar structure has been observed in the small-angle X-ray scattering patterns [55]. Explanations of these morphological features are very important in order to understand the formation mechanism of strain-induced crystals of network polymers and to establish a quantitative crystallization theory in oriented polymer melt.

\section{Conclusion}


On the basis of the development of WAXD patterns and the stress relaxation behaviors of highly-strained rubber samples, we clarified the coexistence of fast and slow processes during the early stage of strain-induced crystallization. The fast process had the time constant in the range of 50 to $200 \mathrm{~ms}$, while the slow process had the time constant in the range of 2.5 to $4.5 \mathrm{~s}$. Crystallization progressed drastically but relative degree of stress relaxation was small in the fast process. On the other hand, though the development of crystallization was relatively small, its correlation with the stress relaxation was apparent during the slow process. Distinct changes of crystallite size and the degree of distortion of crystal lattice with elapsed time were not observed in this study. On the other hand, the time-averaged crystallite size decreased with the increase in average nominal stress in the directions both parallel and perpendicular to the stretching direction. Also the crystal unit cell distorted linearly with the average nominal stress. Some of the features found in the current study may be applicable to understand crystallization of other polymer species from oriented melt. In order to establish a quantitative crystallization theory in oriented polymer melt, explanation of the morphological features found in this series of studies should be important.

\section{Acknowledgment}

The authors are grateful to Dr. M. Kojima of Toyo Tire and Rubber Co., Ltd. for sample preparation. This study was partly supported by a Grant-in Aid for Scientific Research (C), No. 20550187 from Japan Society for the Promotion of Science (to M. Tosaka). The synchrotron radiation experiments were performed at the BL40XU of SPring- 8 with the approval of the Japan Synchrotron Radiation Research Institute (JASRI) (Proposal No. 2010B1215).

\section{References}

1. Storks K H. J Am Chem Soc 1938; 60: 1753-1761.

2. Keller A. Phil Mag 1957; 2: 1171-1178.

3. Till Jr. P H. J Polym Sci 1957; 24: 301-306.

4. Fischer E W. Zeitschrift für Naturforschung 1957; 12a: 753-754.

5. Hoffman J D, Davis G T, Lauritzen J I J. In: Hannay N B, editors. Treatise on Solid State Chemistry, Vol. 3. New York: Plenum Press, 1976. pp. 497-614.

6. Hoffman J D, Miller R L. Polymer 1997; 38: 3151-3212.

7. Armistead K, Goldbeck-Wood G, Keller A. Adv Polym Sci 1992; 100: 221-312.

8. Hikosaka M. Polymer 1987; 28: 1257-1264. 
9. Liedauer S, Eder G, Janeschitz-Kriegl H, Jerschow P, Geymayer W, Ingolic E. Intern Polymer Processing 1993; 8: 236-244.

10. Nogales A, Hsiao B S, Somani R H, Srinivas S, Tsou A H, Balta-Calleja F J, Ezquerra T A. Polymer 2001; 42: 5247-5256.

11. Hayashi Y, Matsuba G, Zhao Y, Nishida K, Kanaya T. Polymer 2009; 50: 2095-2103.

12. Fukushima H, Ogino Y, Matsuba G, Nishida K, Kanaya T. Polymer 2005; 46: 1878-1885.

13. Zhao Y, Matsuba G, Nishida K, Fujiwara T, Inoue R, Polec I, Deng C, Kanaya T. J Polym Sci Part B Polym Phys 2011; 49: 214-221.

14. Yamamoto M, White J L. J Polym Sci Part A-2 1971; 9: 1399-1415.

15. Tosaka M. Macromolecules 2009; 42: 6166-6174.

16. Treloar L R G, The Physics of Rubber Elasticity, Third Edition, Oxford: Clarendon Press, 1975.

17. Flory P J. J Chem Phys 1947; 15: 397-408.

18. Tosaka M, Kohjiya S, Murakami S, Poompradub S, Ikeda Y, Toki S, Sics I, Hsiao B S. Rubber Chem Technol 2004; 77: 711-723.

19. Tosaka M, Murakami S, Poompradub S, Kohjiya S, Ikeda Y, Toki S, Sics I, Hsiao B S. Macromolecules 2004; 37: 3299-3309.

20. Ikeda Y, Yasuda Y, Makino S, Yamamoto S, Tosaka M, Senoo K, Kohjiya S. Polymer 2007; 48: 1171-1175.

21. Ikeda Y, Yasuda Y, Hijikata K, Tosaka M, Kohjiya S. Macromolecules 2008; 41: 58765884.

22. Acken M F, Singer W E, Davey W P. Ind Eng Chem 1932; 24: 54-57.

23. Long J D, Einger W E, Davey W P. Ind Eng Chem 1934; 26: 543-547.

24. Dunning D J, Pennells P J. Rubber Chem Technol 1967; 40: 1381-1393.

25. Mitchell J C, Meier D J. J Polym Sci A-2 1968; 6: 1689-1703.

26. Gent A N. Trans Faraday Soc 1954; 50: 521-533.

27. Luch D, Yeh G S Y. J Polym Sci Polym Phys 1973; 11: 467-486.

28. Valladares D, Yalcin B, Cakmak M. Macromolecules 2005; 38: 9229-9242.

29. Komura M, Nakajima K, Nishi T, Ikehara T, Zhaobin Q. Rubber Chem Technol 2008; 81: 110-120.

30. Tosaka M, Kawakami D, Senoo K, Kohjiya S, Ikeda Y, Toki S, Hsiao B S. Macromolecules 2006; 39: 5100-5105. 
31. Tosaka M, Senoo K, Kohjiya S, Ikeda Y. J Appl Phys 2007; 101: 84909.

32. Toki S, Fujimaki T, Okuyama M. Polymer 2000; 41: 5423-5429.

33. Klug H P, Alexander L E, X-ray Diffraction Procedures for Polycrystalline and Amorphous Materials, 2nd Ed., New York: Wiley-Interscience, 1974.

34. Gent A N, Kawahara S, Zhao J. Rubber Chem Technol 1998; 71: 668-678.

35. Trabelsi S, Albouy P-A, Rault J. Rubber Chem Technol 2004; 77: 303-316.

36. Burfield D R, Tanaka Y. Polymer 1987; 28: 907-910.

37. Tanaka Y. Rubber Chem Technol 2001; 74: 355-375.

38. Nyburg S C. Acta Crystallogr 1954; 7: 385-392.

39. Takahashi Y, Kumano T. Macromolecules 2004; 37: 4860-4864.

40. Wu W. Polym Eng Sci 1979; 19: 391-394.

41. Miyamoto Y, Yamao H, Sekimoto K. Macromolecules 2003; 36: 6462-6471.

42. Toki S, Sics I, Hsiao B S, Tosaka M, Poompradub S, Ikeda Y, Kohjiya S. Macromolecules 2005; 38: 7064-7073.

43. Toki S, Hsiao B S, Kohjiya S, Tosaka M, Tsou A H, Datta S. Rubber Chem Technol 2006; 79: 460-488.

44. Kim H-G, Mandelkern L. J Polym Sci Part A-2 1968; 6: 181-196.

45. Gent A N, Zhang L-Q. J Polym Sci Part 2001; B: Polym. Phys. 39: 811-817.

46. Fujiwara T, Inoue R, Nishida K, Cong D, Polec I, Kanaya T, Ogawa H, Ohta N, Matsuba G. Fiber Prepr Japan 2011; 66: 123.

47. Burfield D R. Polymer 1984; 25: 1823-1826.

48. Andrews E H. Pure Appl Chem 1972; 31: 91-111.

49. Andrews E H. Proc Roy Soc A 1964; 277: 562-570.

50. Shimizu T, Tsuji M, Kohjiya S. Mater Sci Res Int 1998; 4: 117-120.

51. Shimizu T, Tsuji M, Kohjiya S. J Mater Res 1999; 14: 1645-1652.

52. Shimizu T, Tosaka M, Tsuji M, Kohjiya S. Rubber Chem Technol 2000; 73: 926-936.

53. Trabelsi S, Albouy P-A, Rault J. Macromolecules 2003; 36: 7624-7639.

54. Tosaka M, Kohjiya S, Ikeda Y, Toki S, Hsiao B S. Polym J 2010; 42: 474-481.

55. Tosaka M, Toki S, Che J, Rong L, Hsiao B S. J Polym Sci B Polym Phys 2011; 49: 11571162. 


\section{FIGURE CAPTIONS}

Fig. 1 Evolution of WAXD patterns of NR3T at $\alpha_{s}=7$. The rectangles indicate the regions used for the analysis. The arrow points the reflection from stearic acid crystals.

Fig. 2 Development of WAXD intensity of NR3T with elapsed time. The fitting curves are indicated. (a) Comparison among the data obtained from different reflections in the case of $\alpha_{s}=7$. (b) Dependence of the average intensity $I(t)$ on strain ratio, $\alpha_{s}$.

Fig. 3 Time constants (a) $\tau_{f}$ and (b) $\tau_{s}$ of crystallization as a function of strain ratio, $\alpha_{s}$.

Fig. 4 Amplitude of (a) the fast and (b) the slow processes as a function of strain ratio, $\alpha_{s}$.

Fig. 5 Crystallite size $L_{200}$ of the NR samples at $\alpha_{s}=6$ as a function of elapsed time, $t$.

Fig. 6 Average crystallite size (a) $<L_{200}>$ and (b) $<L_{002}>$ as a function of the average nominal stress, and (c) $<L_{200}>$ and $<L_{002}>$ as a function of inverse of the average nominal stress. The solid and broken lines are guides for eyes.

Fig. 7 Time-averaged dimensions of crystal unit cell (a) in the $a$-direction, (b) in the $b$-direction and (c) in the $c$-direction. The solid lines are guides for eyes.

Fig. 8 Stress relaxation behavior of NR3T.

Fig. 9 Time constant $\tau_{\mathrm{x}}$ of the stress relaxation as a function of strain ratio, $\alpha_{\mathrm{s}}$

Fig. 10 Amplitude of the stress relaxation processes (a) $\sigma_{f}$, (b) $\sigma_{s}$ and (c) $\sigma_{x}$.

Fig. 11 Relationship between the amplitudes of crystallization and relative stress relaxation. (a) For the fast crystallization process with time constant $\tau_{f}$ (b) For the slow crystallization process with time constant $\tau_{s}$. The solid line is guide for eyes. 
Fig. 12 Ratio between amplitudes of relative stress relaxation and crystallization. (a) For the fast crystallization process with time constant $\tau_{f}$. (b) For the slow crystallization process with time constant $\tau_{s}$.

Fig. 13 Contribution of strain-induced crystallization to the total degree of stress relaxation. 


\section{TABLES}

Table 1 Recipes of vulcanized rubber samples in part.

\begin{tabular}{|c|c|c|c|c|c|c|}
\hline Sample & Rubber & \multicolumn{2}{|c|}{ Stearic acid } & $\mathrm{ZnO}$ & $\mathrm{CBS}^{\mathrm{c}}$ & Sulfi \\
\hline NR1T & $100^{\mathrm{a}}$ & \multicolumn{2}{|l|}{2} & 1 & 3 & 4.5 \\
\hline NR3T & $100^{\mathrm{a}}$ & \multicolumn{2}{|l|}{2} & 1 & 1.5 & 2.25 \\
\hline NR5T & $100^{\mathrm{a}}$ & \multicolumn{2}{|l|}{2} & 1 & 0.75 & 1.12 \\
\hline IR3T & $100^{\mathrm{b}}$ & \multicolumn{2}{|l|}{2} & 1 & 1.5 & 2.25 \\
\hline IR5T & $100^{\mathrm{b}}$ & \multicolumn{2}{|l|}{2} & 1 & 0.75 & 1.12 \\
\hline \multicolumn{7}{|l|}{${ }^{\mathrm{a}} \mathrm{RSS}$ \#3 } \\
\hline \multicolumn{7}{|l|}{${ }^{\mathrm{b}} \mathrm{IR} 2200$} \\
\hline \multicolumn{7}{|c|}{${ }^{\mathrm{c}} N$-cyclohexyl-2-benzothiazole sulfenamide } \\
\hline Sample & Curing $\mathrm{t}$ & $\min )$ & $v \times 1$ & (mol) & $\left.\mathrm{m}^{3}\right)$ & \\
\hline NR1T & 20 & & 2.44 & & & \\
\hline NR3T & 25 & & 1.95 & & & \\
\hline NR5T & 35 & & 1.48 & & & \\
\hline IR3T & 35 & & 1.76 & & & \\
\hline IR5T & 50 & & 1.49 & & & \\
\hline
\end{tabular}

